\title{
Neoadjuvant concurrent chemoradiotherapy with and without hyperthermia in retroperitoneal and intra-abdominal sarcomas: Feasibility, efficacy, toxicity and long-term outcome
}

\author{
Alexander Willner \\ University of Erlangen-Nuremberg \\ Katja Fechner \\ Universitätsklinikum Erlangen \\ Abbas Agaimy \\ Universitätsklinikum Erlangen \\ Florian Haller \\ Universitätsklinikum Erlangen \\ Markus Eckstein \\ Universitätsklinikum Erlangen
}

Oliver Ott

Universitätsklinikum Erlangen

Florian Putz

Universitätsklinikum Erlangen

Udo Gaipl

Universitätsklinikum Erlangen

Stephan Kersting

Universitätsklinikum Erlangen

Norbert Meidenbauer

Universitätsklinikum Erlangen

Robert Grützmann

Universitätsklinikum Erlangen

Rainer Fietkau

Universitätsklinikum Erlangen

Sabine Semrau ( $\square$ sabine.semrau@uk-erlangen.de)

Universitätsklinikum Erlangen

Research Article 
Keywords: Retroperitoneal, sarcoma, neoadjuvant, chemoradiotherapy

Posted Date: February 25th, 2021

DOI: https://doi.org/10.21203/rs.3.rs-134064/v1

License: (c) (1) This work is licensed under a Creative Commons Attribution 4.0 International License. Read Full License 


\section{Abstract}

Background Retroperitoneal (RPS) and intra-abdominal sarcomas (IAS) are associated with poor local and abdominal tumor control. Yet, the benefit of preoperative radio- or chemotherapy alone for these entities currently is unclear. Moreover, as intermediate- and high-grade sarcomas have a tendency for early metastasis, exploration of neoadjuvant strategies is of high importance. This analysis reports the results of our 20-year single-institution experience with preoperative neoadjuvant concurrent chemoradiation.

Methods From 2000-2019, 27 patients with intermediate- or high-grade RPS or IAS (12 dedifferentiated liposarcoma, 10 leiomyosarcoma, 5 others) were treated with radiotherapy (median dose: $50.4 \mathrm{~Gy}$; range 45-75 Gy) and two cycles of chemotherapy (doxorubicin $50 \mathrm{mg} / \mathrm{m}^{2} \mathrm{BSA} / \mathrm{d} 3 \mathrm{q} 28$ and ifosfamide $1.5 \mathrm{~g} / \mathrm{m} 2$ $\mathrm{BSA} / \mathrm{d} 1-5 \mathrm{q} 28$ ) in neoadjuvant intention. Chemotherapy consisted of doxorubicin alone in two cases and ifosfamide alone in one case. Fifteen patients (56\%) received deep regional hyperthermia additionally.

Results The median follow-up time was 53 months ( \pm 56.7 months). $92 \%$ of patients received two cycles of chemotherapy as planned and $92 \%$ underwent surgery. At 5 and 10 years, abdominal-recurrence-freesurvival was $74.6 \%( \pm 10.1 \%)$ and $66.3 \%( \pm 11.9 \%)$, distant-metastasis-free-survival was $67.2 \%( \pm 9.7 \%)$ and $59.7 \%( \pm 11.1 \%)$, and overall-survival was $60.3 \%( \pm 10.5 \%)$ and $60.3 \%( \pm 10.5 \%)$, respectively. CTC grade III and IV toxicities were leukocytopenia (85\%), thrombocytopenia (33\%) and anemia (11\%). There were no treatment-related deaths.

Conclusions Neoadjuvant chemoradiotherapy with and without hyperthermia for retroperitoneal and intraabdominal sarcoma is feasible and provided high local control of intermediate - and high-grade sarcoma.

\section{Background}

Retroperitoneal sarcoma (RPS) and intra-abdominal sarcomas (IAS) are rare and challenging oncologic entities. Liposarcoma and leiomyosarcoma are the main histologic types encountered, with high-grade histology being present in half of all cases (1-3). Although surgical resection is the mainstay of treatment for cases without distant metastasis, most patients ultimately develop intra-abdominal recurrences after resection alone (4), which is the rationale for additive radiation treatment (RT). Retrospective case series results suggest that postoperative radiotherapy (PORT) may provide increase local tumor control (5-7). There are, however, limitations for the implementation of PORT, such as problems in defining the postoperative volume to be irradiated after resection and the risk of higher long-term gastrointestinal toxicity, as abdominal organs settle back into their regular positions after surgery (8). On the other hand, preoperative radiotherapy has the advantage of a more straightforward definition of the clinical target volume and the reduction of late side effects in patients with soft-tissue sarcoma of the limbs (9). The EORTC-STRASS trial investigated the benefits of preoperative radiotherapy for RPS and showed that neoadjuvant radiotherapy is well-tolerated but does not improve abdominal-recurrence-free-survival (AFRS) (10). Subgroup analyses revealed that preoperative radiotherapy significantly improved AFRS in 
low-grade sarcoma and liposarcoma, but not in high- and intermediate-grade sarcoma, suggesting that the different grades of RPS require a differentiated treatment approach according to tumor grade.

These findings raise the important question whether the addition of chemotherapy (CT) and hyperthermia $(\mathrm{HT})$ to radiotherapy might result in improvement of intra-abdominal control rates. To explore this question, this retrospective analysis was designed to treatment outcome of RPS and IAS patients who received combined chemoradiotherapy (CRT) at the Department of Radiation Oncology, University Hospital of Erlangen. In recent years, neoadjuvant concurrent chemoradiotherapy (CCRT) has been expanded to additionally include hyperthermia in selected cases (11).

\section{Methods}

\section{Patient characteristics:}

All data on the course of disease and follow-up of all sarcoma cases entered in our hospital database since 2000 were retrospectively evaluated (see Table 1). The cut-off date for analysis was August $31^{\text {st }}$, 2019. The management of each case was discussed primarily in a multidisciplinary team setting before and after treatment, informed consent was obtained from all participants. According to the protocol, biopsy followed by neoadjuvant concurrent chemoradiotherapy was performed in all cases with confirmed FNCLCC grade G2 or G3 tumors. Neoadjuvant chemotherapy alone was not performed. Exclusion criteria for neoadjuvant CCRT were ECOG $>1$, intestinal passage obstruction and/or age ${ }^{3} 80$ years. Patients with a high risk of severe renal failure following radiotherapy with or without subsequent nephrectomy were also excluded. This was determined by MAG3 scans (isotope nephrography) for the individual assessment of left and right renal function. Histological diagnosis was based on the World Health Organization (WHO) classification of soft tissue tumors valid at initial biopsy diagnosis. Tumor grading was performed according to the French grading system (FNCLCC).

\section{Treatment}

\section{Radiotherapy}

The radiation techniques used were multi-field 3D RT with a dose prescribed to the ICRU50 reference point until 2012, and intensity-modulated radiation therapy (IMRT) or volumetric modulated arc therapy (VMAT) in subsequent years. Doses were generally delivered in fractions of $1.8 \mathrm{~Gy}$, five times a week. However, three patients received fractions of $1.5 \mathrm{~Gy}$ instead. Four patients treated before 2010 had a treatment protocol specifying a seven-day treatment break in week 3 (see Figure 1).

\section{Chemotherapy}

Twenty-four patients received a combination regimen of doxorubicin $\left(50 \mathrm{mg} / \mathrm{m}^{2} / \mathrm{d}\right.$ on day 3$)$ plus ifosfamide $\left(1.5 \mathrm{~g} / \mathrm{m}^{2} / \mathrm{d}\right.$ on days $\left.1-5\right)$ in weeks 1 and 5 (if they had sufficient cardiac and renal function) with appropriate antiemetic therapy (HT3 antagonist), hydration and cystitis prophylaxis. Three patients 
had comorbidities at baseline that required chemotherapy dose reduction. The first cycle of chemotherapy was started in parallel with radiation. Granulocyte colony-stimulating factor (G-CSF) was not administered prophylactically but was given to patients with leukocytopenia (count $<1500 / \mu \mathrm{L}$ ). To receive the second cycle of chemotherapy in week 5 of radiotherapy, patients had to have a leukocyte count greater than $3000 / \mu \mathrm{L}$ and a platelet count greater than $100,000 / \mu \mathrm{L}$. If not, the second cycle was postponed by a maximum of 2 weeks or was not performed in case of inadequate restitution of hematopoiesis. Postoperative or consolidating chemotherapy was not performed in patients with inoperable disease.

\section{Hyperthermia:}

From 2004 on, regional hyperthermia was performed with a SD 2000 hyperthermia system (BSD Medical/Pyrexar, Salt Lake City, UT, USA) with or without non-invasive MR thermometry with 1-2 treatments/week. The target temperature was $40-44^{\circ} \mathrm{C}$ for 60 minutes per treatment.

\section{Surgical resection:}

En bloc resection of the tumor was to be carried out 6-10 weeks after the end of chemoradiotherapy if possible. The treatment regimen is illustrated in Figure 1. Additional pictures detailling the treatment regimen are shown in Figure 2.

\section{Follow-up and statistics:}

The feasibility of chemotherapy was verified based on data in the patient treatment records and pharmacy records. The performance of radiotherapy was confirmed using the MOSAIQ Record \& Verify System. Treatment toxicity was graded as per the Common Terminology Criteria for Adverse Events (CTCAE) version 4.0 (12). Postoperative complications were assessed according to data in the electronic patient records. After the treatment, patients were scheduled for quarterly clinical and semi-annual computed tomography (CT) follow-up assessments. All patients without reported incidents were contacted by phone on the follow-up assessment date. Two patients were lost to follow-up. The data from their last clinical visits were included in the analysis.

Endpoints were abdominal-recurrence-free-survival (ARFS, defined as the time to abdominal relapse after R0, R1 or R2 resection), distant-metastasis-free-survival (DMFS), disease-free-survival (DFS) and overallsurvival (OS).

Statistical analysis was performed using SPSS Statistics Version 26. Survival analysis was performed by means of the Kaplan-Meier method and the logrank test as well as Cox's regression analysis. Differences of $p<0.05$ were defined as statistically significant.

\section{Results}

\section{Patients:}


Of the 27 patients included in the analysis, three ultimately received definitive treatment because resectability was not archieved: persistent inoperability was confirmed by the multidisciplinary team based on the results of repeat imaging studies after the patients had received 50.4 Gy of radiation. Twenty-four patients were classified as potentially resectable and underwent surgery during the further course. Characteristics of the patient population are summarized in Table 1. Of the 27 patients, 26 (96\%) had intermediate and high-grade sarcomas and one (female) had a single-site, multi-recurrent low-grade liposarcoma. The histological subtype was dedifferentiated liposarcoma in 12 cases ( 7 intermediate grade and 5 high grade), leiomyosarcoma in 10 patients ( 5 intermediate grade and 5 high grade) and 4 other subtypes (4 high grade).

\section{Treatment feasibility:}

All patients received the intended prescription dose of 45-55 Gy in the neoadjuvant setting and 60-65 Gy in the definitive setting. The median radiation dose (with standard deviation, SD) was $54.4 \pm 4.1 \mathrm{~Gy}$ in the neoadjuvant setting and $65.0 \pm 8.7 \mathrm{~Gy}$ in the definitive setting (see Table 2).

Concomitant chemotherapy consisting of doxorubicin plus ifosfamide was administered to 24 patients. Of these patients, $7(29 \%)$ received $>80 \%$ of the planned dose of ifosfamide, while $11(46 \%)$ received $>50 \%$ and $6(25 \%)$ received $30 \%-50 \%$ of the total dose of $15 \mathrm{~g} / \mathrm{m}^{2}$ of ifosfamide planned for the two cycles. For doxorubicin, 20 (83\%) of the patients received more than $80 \%$ of the planned dose of $100 \mathrm{mg} / \mathrm{m}^{2}$ over two cycles, and the remaining four patients received $50 \%$ to $80 \%$ of the target dose. Chemotherapy was administered according to protocol in 24 cases (89\%).

Due to age $>80$ years, two patients received weekly full-dose doxorubicin monotherapy for at least 5 cycles (to a total dose of $75 \mathrm{mg} / \mathrm{m}^{2}$ ). In the remaining case, a patient pretreated with doxorubicin received ifosfamide parallel to radiotherapy at a dose of $1.5 \mathrm{~g} / \mathrm{m}^{2} / \mathrm{d}$ on days $1-5$ but could not receive the second cycle due to severe deterioration of his general health.

Hyperthermia treatment (HT) was administered parallel to RCT in $15(56 \%)$ patients, who received a median of $5 \mathrm{HT}$ treatments.

Of the patients who received neoadjuvant therapy, $16(67 \%)$ were classified as R0 after en-bloc resection (see Table 2), 6 (25\%) as R1. The resection status of $2(8 \%)$ could not be determined due to missing data at the time of the analysis.

\section{Toxicity profile:}

The most common adverse events of chemoradiotherapy were hematological side effects (see Table 3 ). Surgical resection could be performed at the planned time in all cases. The most common AEs were radiation dermatitis (19\%, none CTCAE grade 3 or 4 ) and diarrhea (6\%). Eight patients (26\%) experienced nausea throughout the treatment. Postoperative complications were acute renal failure $(n=1)$, burst abdomen $(n=1)$, anastomotic leakage $(n=1)$, and cerebellitis $(n=1) 3$ weeks after surgery. The case of cerebellitis was determined to be autoimmune and resolved spontaneously within a few weeks. 


\section{Efficacy:}

\section{Abdominal-recurrence-free-survival (ARFS)}

The rate of abdominal recurrence-free survival was $95.8 \%( \pm 4.1 \%)$ at one-year follow-up, $74.6 \%( \pm 10.1 \%)$ at three years, $74.6 \%( \pm 10.1 \%)$ at five years, and $66.3 \%( \pm 11.9 \%)$ at ten years (see Figure 3$)$. Tumors $<10 \mathrm{~cm}$ vs. $\geq 10 \mathrm{~cm}$ in diameter showed differences in the length of ARFS, yet they were not significant ( $p=0.076$, univariate analysis). ARFS rates were slightly better in $\mathrm{G} 2$ tumors, compared to $\mathrm{G} 3$ tumors, in patients who received more than six hyperthermia treatment sessions and younger patients. Still, the differences were not significant (see Table 4). In multivariate Cox's regression analysis, none of the variables was found to be an independent prognostic factor but no conclusions should be drawn from this finding in view of the low number of events. In case of recurrence $(n=15)$, the decision to perform chemotherapy $(n=4)$, repeat chemoradiotherapy $(n=1)$, radiotherapy $(n=1)$, surgical resection $(n=5)$ or palliative treatment $(n=4)$ was made on a case-by-case basis. Local recurrence appeared infield in 3 patients and outfield in 3 patients.

\section{Distant-metastasis-free-survival (DMFS):}

Ten patients developed distant metastases, which initially occurred in the lungs in six cases, in the bone in three, and in the duodenum in one case. In $90 \%$ of cases, the metastases developed within 2 years of the start of treatment. The DMFS rate was $67.2 \%( \pm 9.7 \%)$ at 3 years, $67.2 \%( \pm 9.7 \%)$ at 5 years, and $59.7 \%$ $( \pm 11.1 \%)$ at 10 years. The difference in DMFS between recurrences and primary tumors was noticeable: $56 \%$ of patients with recurrent tumors developed further recurrences compared to $32 \%$ of those with primary tumors. In the case of DMFS, no independent predictors could be identified by multivariate analysis either in context of a low number of events. There was no difference in DMFS between patients with and without hyperthermia treatment.

\section{Disease-free-survival (DFS):}

Disease-free survival was $48.1 \%( \pm 10.8 \%)$ at 3 years, $48.1 \%( \pm 10.8 \%)$ at 5 years, and $36.1 \%( \pm 11.0 \%)$ at 10 years. No confounding factors could be identified. Eleven patients received second-line therapy, consisting of surgery for recurrent disease in five cases, radiotherapy in one case, and systemic therapy in five cases. Four patients received palliative treatment.

\section{Overall-survival (OS):}

On the cut-off date for analysis, 16 patients (59\%) were still alive and $2(7 \%)$ were lost to follow-up. Overall survival was $70.4 \%( \pm 9.5 \%)$ at 3 years, $60.3 \%( \pm 10.5 \%)$ at 5 years, and $60.3 \%( \pm 10.5 \%)$ at 10 years (see Figure 4). Over the long term, women and patients with $\mathrm{G} 2$ tumors had better overall survival, but the difference was not significant. Recurrent disease patients who were diagnosed early enough that the recurrent tumors could be treated survived a median of 40 months. 


\section{Discussion}

Concurrent chemoradiotherapy protocols combining chemotherapy with radiation treatment have now gained acceptance as modalities for treating a wide range of locally advanced solid tumors in the neoadjuvant setting (13-15). Intra-abdominal and retroperitoneal sarcomas pose similar challenges to surgeons regarding local resectability and recurrence rates after surgical resection alone. Nevertheless, neoadjuvant chemoradiotherapy is not a standard treatment for these tumor entities, especially after the negative results of the EORTC-STRASS study (10). We administer CCRT to patients with high- or intermediate-grade sarcomas on a case-by-case decision basis. Our experience is based on the treatment of sarcomas of the trunk and extremities. As early as 1999, Sauer et al. demonstrated in 22 patients initially classified as inoperable that secondary resectability could be achieved with neoadjuvant chemoradiotherapy (16). However, almost half of their patients developed high-grade toxicities, and one patient died (4\%). Given the poor prognosis of sarcoma patients without resection, treatment toxicity was rated as acceptable because it achieved resectability. A later study of neoadjuvant CRT and surgery in 53 soft tissue sarcoma patients by Stubbe et al. resulted in a high long-term local recurrence rate of $90 \%$ (11) and one postoperative death (2\%). Fourteen patients in their population had retroperitoneal sarcoma. The toxicity of treatment of RPS patients was not higher than that of patients with soft-tissue sarcoma of the extremities. Therefore, the concept was further established in the following years. We now report the results of the treatment of 27 cases of retroperitoneal and intra-abdominal sarcoma. This constitutes the most extensive published series of RPS/IAS patients treated with neoadjuvant concurrent chemoradiotherapy.

Our analysis of the data shows that the side effects of treatment are manageable, even when the combination of radiotherapy and chemotherapy is administered in a single-center setting directed by one department. Our treatment concept includes intensive supportive therapy with parenteral nutrition and antiemetic therapy as needed as well as early stimulation therapy in case of hematological toxicity. The findings of the RTOG-9514 study highlight the importance of supportive treatment (17). Five percent of deaths observed in RTOG-9514 occurred in patients treated using a multicenter and multimodal approach. According to our analysis, hematological and gastrointestinal toxicities were the most critical factors, but they were well controlled by parenteral nutrition, and no increase in postoperative morbidity occurred.

Analysis of our population showed that neoadjuvant CCRT can achieve excellent local control of both surgically resected and inoperable RPS/IAS. Regarding the EORTC-STRASS study (10), the 5-year local control rate was $75 \%$ in the present study compared to only $47.6 \%$ in the EORTC-STRASS study. Moreover, our patient population had a less favorable prognosis as it was composed almost entirely of patients with high- or intermediate-grade tumors, whereas $34.6 \%$ of patients included in the STRASS study had low-grade sarcomas. Nevertheless, the benefit of neoadjuvant concurrent chemoradiotherapy must be evaluated in conjunction with the results of other studies. Data on this subject is sparse. For example, Gronchi et al. $(18,19)$ achieved a local control rate of $63 \%$ with a neoadjuvant CCRT regimen consisting of radiotherapy up to a total dose of $50.4 \mathrm{~Gy}$ and high dose ifosfamide chemotherapy. Their treatment 
protocol was also feasible. They also included patients with low-grade RPS but required a minimum tumor size of $5 \mathrm{~cm}$ in diameter. The latter size specification was not always met in our study. Studies of neoadjuvant chemotherapy alone are also rare (20).

Encouraged by the findings of Issels et al. (21), we administered hyperthermia treatment in addition to chemotherapy in almost half of our patients. In the end, we could not detect any significant difference between the patients who received hyperthermia and those who did not. This may be due to several factors. The main reason might be the small size of the sample, which resulted in reduced statistical power. Furthermore, it cannot be excluded that chemoradiotherapy alone might have already had a sufficient local therapeutic effect. Finally, it can be concluded that hyperthermia did not result in increased toxicity, so adding regional hyperthermia to the regimen as described by Issels et al. (22) is not problematic.

\section{Summary}

In conclusion, the neoadjuvant concurrent chemoradiotherapy regimen established by us for selected retroperitoneal and intra-abdominal sarcoma patients is feasible and does not increase postoperative morbidity. In cases with and without surgical tumor resection, this approach achieved excellent local control rates, better than those obtained with radiotherapy alone. This constitutes an important signal that combined chemoradiation might be superior to radiotherapy alone in retroperitoneal and intraperitoneal sarcomas. The rate of distant metastasis of high- and intermediate-grade sarcomas remains high and needs further improvement.

\section{Declarations}

\section{Ethics approval and consent to participate}

Each patient has agreed to the treatment and the subsequent use of the data for research purposes prior to the start of treatment in a separate treatment contract, which is available. Therefore, the Ethics Committee of the Friedrich-Alexander-University Erlangen-Nuremberg waived an additional approval on the use of data. Treatment and data collection had been performed in accordance with the Declaration of Helsinki.

\section{Consent for publication}

Informed consent was obtained from all participants or, if participants were under 18 , from a parent and/or legal guardian.

\section{Availability of data and materials}

The datasets used and/or analysed during the current article are available from the corresponding author on reasonable request, please contact the corresponding author (Sabine.Semrau@uk-erlangen.de). 


\section{Competing interests}

The authors declare that they do not have any competing interests.

\section{Funding}

Not applicable.

\section{Authors' contributions}

Willner, Analysis and interpretation of the patient data regarding their tumor and treatment characteristics, statistical analysis, interpretation of analysis and Alexander writing the manuscript.

Fechner, Collection of data, therapy of patients, concept of analysis and approval of the Katja manuscript

Agaimy, Pathological examination and approval of the manuscript Abbas

Haller, $\quad$ Pathological examination and approval of the manuscript Florian

Eckstein, Pathological examination and approval of the manuscript Markus

Ott, OJ Planning of radiotherapy, planning of hyperthermia and approval of the manuscript

Putz, Florian Therapy of patients, statistical supervision and approval of the manuscript

Gaipl, US Concept of hyperthermia and approval of the manuscript

Kersting, Operative patient treatment and approval of the manuscript

Stephan

Meidenbauer, Therapy of patients and approval of the manuscript

Norbert

Grützmann, Operative patient treatment and approval of the manuscript

Robert

Fietkau, Concept of therapy, therapy of patients

Rainer

Semrau, Collection of data, therapy of patients, concept of analysis and approval of the Sabine manuscript

\section{Acknowledgements}

This article was published to meet the requirements of the Friedrich-Alexander-University ErlangenNuremberg (FAU) for obtaining the German doctoral degree in medicine ("Dr. med."). 


\section{References}

Cancer facts and figures 2017. https://www.cancer.org/research/cancer-facts-statistics/all-cancer-factsfigures/cancer-facts-figures-2017.html

Accessed 25.09.2020

Cancer facts and figures 2019. https://www.cancer.org/research/cancer-facts-statistics/all-cancer-factsfigures/cancer-facts-figures-2019.html

Accessed 25.09.2020

German guidelines for soft tissue sarcoma 2019.

https://www.onkopedia.com/de/onkopedia/guidelines/weichgewebssarkome-maligneweichgewebstumoren-des-erwachsenen/@@guideline/html/index.html

Accessed 25.09.2020

Gronchi A, Strauss DC, Miceli R, et al. Variability in Patterns of Recurrence After Resection of Primary Retroperitoneal Sarcoma (RPS): A Report on 1007 Patients From the Multi-institutional Collaborative RPS Working Group. Ann Surg. 2016;263(5):1002-1009. doi:10.1097/SLA.0000000000001447

Jakob, J., Lesluyes, T., Simeonova-Chergou, A. et al. Impact of preoperative treatment on the CINSARC prognostic signature: translational research results from a phase 1 trial of the German Interdisciplinary Sarcoma Group (GISG 03). Strahlenther Onko/ 196, 280-285 (2020). https://doi.org/10.1007/s00066019-01543-5

Kirste, S., Landenberger, N., Scholber, J. et al. Retroperitoneal soft tissue sarcoma: low-dose neoadjuvant radiation therapy followed by surgery with or without intraoperative radiotherapy and adjuvant radiation therapy. Strahlenther Onko/ 195, 558-565 (2019). https://doi.org/10.1007/s00066-019-01464-3

(7) 
Dunst J. Prä- oder postoperative Strahlentherapie bei retroperitonealen Sarkomen unverzichtbar [Pre- or postoperative radiotherapy essential for the treatment of retroperitoneal sarcomas]. Strahlenther Onkol. 2016;192(11):820-822. doi:10.1007/s00066-016-1042-4

Gilbeau L, Kantor G, Stoeckle E, et al. Surgical resection and radiotherapy for primary retroperitoneal soft tissue sarcoma. Radiother Oncol. 2002;65(3):137-143. doi:10.1016/s0167-8140(02)00283-9

O'Sullivan B, Davis AM, Turcotte R, et al. Preoperative versus postoperative radiotherapy in soft-tissue sarcoma of the limbs: a randomised trial. Lancet. 2002;359(9325):2235-2241. doi:10.1016/S01406736(02)09292-9

Bonvalot S, Gronchi A, Le Péchoux C, Swallow CJ, Strauss D, Meeus P, van Coevorden F, Stoldt S, Stoeckle E, Rutkowski P, Rastrelli M, Raut CP, Hompes D, De Paoli A, Sangalli C, Honoré C, Chung P, Miah A, Blay JY, Fiore M, Stelmes JJ, Dei Tos AP, Baldini EH, Litière S, Marreaud S, Gelderblom H, Haas RL. Preoperative radiotherapy plus surgery versus surgery alone for patients with primary retroperitoneal sarcoma (EORTC62092: STRASS): a multicentre, open-label, randomised, phase 3 trial. Lancet Oncol. 2020 Sep 14:S14702045(20)30446-0. doi: 10.1016/S1470-2045(20)30446-0. Epub ahead of print. PMID: 32941794.

Stubbe F, Agaimy A, Ott O, et al. Effective local control of advanced soft tissue sarcoma with neoadjuvant chemoradiotherapy and surgery: A single institutional experience. Cancer Radiother. 2016;20(1):6-13. doi:10.1016/j.canrad.2015.05.032

Common Terminology Criteria for Adverse Events 4.0. https://www.eortc.be/services/doc/ctc/CTCAE_4.03_2010-06-14_QuickReference_5x7.pdf Accessed 25.09.2020

Sauer R, Becker H, Hohenberger W, et al. Preoperative versus postoperative chemoradiotherapy for rectal cancer. N Engl J Med. 2004;351(17):1731-1740. doi:10.1056/NEJMoa040694 
Sauer R, Liersch T, Merkel S, et al. Preoperative versus postoperative chemoradiotherapy for locally advanced rectal cancer: results of the German CAO/ARO/AIO-94 randomized phase III trial after a median follow-up of 11 years. J Clin Oncol. 2012;30(16):1926-1933. doi:10.1200/JC0.2011.40.1836

Shapiro J, van Lanschot JJB, Hulshof MCCM, et al. Neoadjuvant chemoradiotherapy plus surgery versus surgery alone for oesophageal or junctional cancer (CROSS): long-term results of a randomised controlled trial. Lancet Oncol. 2015;16(9):1090-1098. doi:10.1016/S1470-2045(15)00040-6

Sauer R, Schuchardt U, Hohenberger W, Wittekind C, Papadopoulos T, Grabenbauer GG, Fietkau R. Neoadjuvante Radiochemotherapie von Weichteilsarkomen. Optimierung der lokalen funktionserhaltenden Tumorkontrolle [Neoadjuvant radiochemotherapy in soft tissue sarcomas. Optimization of local functional tumor control]. Strahlenther Onkol. 1999 Jun;175(6):259-66. German. doi: 10.1007/BF02743576. PMID: 10392166.

Kraybill WG, Harris J, Spiro IJ, et al. Phase II study of neoadjuvant chemotherapy and radiation therapy in the management of high-risk, high-grade, soft tissue sarcomas of the extremities and body wall: Radiation Therapy Oncology Group Trial 9514. J Clin Oncol. 2006;24(4):619-625. doi:10.1200/JC0.2005.02.5577

Gronchi A, De Paoli A, Dani C, et al. Preoperative chemo-radiation therapy for localised retroperitoneal sarcoma: a phase I-II study from the Italian Sarcoma Group. Eur J Cancer. 2014;50(4):784-792. doi:10.1016/j.ejca.2013.11.021

De Sanctis R, Giordano L, Colombo C, De Paoli A, Navarria P, Sangalli C, Buonadonna A, Sanfilippo R, Bertola G, Fiore M, Marrari A, Navarria F, Bertuzzi A, Casali PG, Basso S, Santoro A, Quagliuolo V, Gronchi A. Long-term Follow-up and Post-relapse Outcome of Patients with Localized Retroperitoneal Sarcoma Treated in the Italian Sarcoma Group-Soft Tissue Sarcoma (ISG-STS) Protocol 0303. Ann Surg Oncol. 2017 Dec;24(13):3872-3879. doi: 10.1245/s10434-017-6105-y. Epub 2017 Oct 17. PMID: 29043525.

Gronchi A, Palmerini E, Quagliuolo V, Martin Broto J, Lopez Pousa A, Grignani G, Brunello A, Blay JY, Tendero O, Diaz Beveridge R, Ferraresi V, Lugowska I, Merlo DF, Fontana V, Marchesi E, Braglia L, Donati DM, Palassini E, Bianchi G, Marrari A, Morosi C, Stacchiotti S, Bagué S, Coindre JM, Dei Tos AP, Picci P, 
Bruzzi P, Casali PG. Neoadjuvant Chemotherapy in High-Risk Soft Tissue Sarcomas: Final Results of a Randomized Trial From Italian (ISG), Spanish (GEIS), French (FSG), and Polish (PSG) Sarcoma Groups. J Clin Oncol. 2020 Jul 1;38(19):2178-2186. doi: 10.1200/JC0.19.03289. Epub 2020 May 18. PMID: 32421444.

Issels RD, Lindner LH, Verweij J, et al. Neo-adjuvant chemotherapy alone or with regional hyperthermia for localised high-risk soft-tissue sarcoma: a randomised phase 3 multicentre study. Lancet Oncol. 2010;11(6):561-570. doi:10.1016/S1470-2045(10)70071-1

Issels RD, Lindner LH, Verweij J, et al. Effect of Neoadjuvant Chemotherapy Plus Regional Hyperthermia on Long-term Outcomes Among Patients With Localized High-Risk Soft Tissue Sarcoma: The EORTC 62961-ESHO 95 Randomized Clinical Trial [published correction appears in JAMA Oncol. 2018 Apr 1;4(4):590]. JAMA Oncol. 2018;4(4):483-492. doi:10.1001/jamaoncol.2017.4996

\section{Tables}

(1) 


\begin{tabular}{|c|c|}
\hline Table 1 & Patient and tumor characteristics \\
\hline Patients: & $N=27(100 \%)$ \\
\hline \multicolumn{2}{|l|}{ Gender } \\
\hline Male & $14(52 \%)$ \\
\hline Female & $13(48 \%)$ \\
\hline Age at first diagnosis & 61 years (range $24-80$ years) \\
\hline Median follow-up & 53 months \\
\hline Tumor & $\mathbf{N}(\%)$ \\
\hline Primary tumor & $18(67 \%)$ \\
\hline Recurrent tumor & $9(33 \%)$ \\
\hline \multicolumn{2}{|l|}{ Histological type } \\
\hline Liposarcoma & $13(48 \%)$ \\
\hline Leiomyosarcoma & $10(37 \%)$ \\
\hline Pleomorphic sarcoma & $4(15 \%)$ \\
\hline \multicolumn{2}{|l|}{ Grading } \\
\hline G1 & $1(4 \%)$ \\
\hline G2 & $12(44 \%)$ \\
\hline G3 & $14(52 \%)$ \\
\hline \multicolumn{2}{|l|}{ Tumor stage } \\
\hline $\mathrm{T} 1$ & $1(4 \%)$ \\
\hline T2 & $20(74 \%)$ \\
\hline T3 & $2(7 \%)$ \\
\hline $\mathrm{T} 4$ & $4(15 \%)$ \\
\hline \multicolumn{2}{|l|}{ Nodal stage } \\
\hline NO & $27(100 \%)$ \\
\hline \multicolumn{2}{|l|}{ UICC 2017} \\
\hline IB & $1(4 \%)$ \\
\hline II & $1(4 \%)$ \\
\hline IIIA & $18(67 \%)$ \\
\hline
\end{tabular}




\begin{tabular}{|ll|} 
IIIB & $7(26 \%)$ \\
\hline Mean tumor diameter & $122 \mathrm{~mm}$ (range $35-350 \mathrm{~mm})$ \\
\hline
\end{tabular}

Table 1: Patient and tumor characteristics

(2) 


\begin{tabular}{|c|c|}
\hline Table 2 & Treatment specifications \\
\hline Radiotherapy & $N(\%)$ \\
\hline \multicolumn{2}{|l|}{ Method } \\
\hline Intensity-modulated & $8(30 \%)$ \\
\hline 3D & $19(70 \%)$ \\
\hline \multicolumn{2}{|l|}{ Fraction size } \\
\hline $1.5 \mathrm{~Gy}$ & $3(11 \%)$ \\
\hline $1.8 \mathrm{~Gy}$ & $3(11 \%)$ \\
\hline $2.0 \mathrm{~Gy}$ & $21(78 \%)$ \\
\hline Boost & $19(70 \%)$ \\
\hline \multicolumn{2}{|l|}{ Fractionation in overall dose } \\
\hline$<50$ Gy & $1(4 \%)$ \\
\hline $50-54.9$ Gy & $10(37 \%)$ \\
\hline $55-60$ Gy & $14(52 \%)$ \\
\hline$>60$ Gy & $2(7 \%)$ \\
\hline \multicolumn{2}{|l|}{ Treatment delay } \\
\hline$<5 d$ & $23(85 \%)$ \\
\hline$\geq 5 d$ & $4(15 \%)$ \\
\hline Chemotherapy & $\mathbf{N}(\%)$ \\
\hline \multicolumn{2}{|l|}{ Type of chemotherapy } \\
\hline Doxorubicin + ifosfamide & $24(89 \%)$ \\
\hline Ifosfamide only & $1(4 \%)$ \\
\hline Doxorubicin only & $2(7 \%)$ \\
\hline \multicolumn{2}{|l|}{ Dose of chemotherapy } \\
\hline$<50 \%$ of planned dose & $7(26 \%)$ \\
\hline$\geq 50 \%$ of planned dose & $20(64 \%)$ \\
\hline \multicolumn{2}{|l|}{ Cycles of chemotherapy } \\
\hline$<2$ cycles & $2(7 \%)$ \\
\hline$\geq 2$ cycles & $25(93 \%)$ \\
\hline
\end{tabular}




\begin{tabular}{|c|c|}
\hline Hyperthermia & $\mathbf{N}(\%)$ \\
\hline Hyperthermia & $15(56 \%)$ \\
\hline$<5$ treatments & $8(53 \%)$ \\
\hline$\geq 5$ treatments & 7 (47\%) \\
\hline
\end{tabular}

Table 2: Treatment specifications

(3) 


\begin{tabular}{|c|c|}
\hline Table 3 & Treatment toxicity \\
\hline \multicolumn{2}{|l|}{ Toxicity } \\
\hline Leukocytopenia & $26(96 \%)$ \\
\hline Grade 3 & $6(22 \%)$ \\
\hline Grade 4 & $17(63 \%)$ \\
\hline Thrombocytopenia & $18(67 \%)$ \\
\hline Grade 3 & $3(11 \%)$ \\
\hline Grade 4 & $6(22 \%)$ \\
\hline Anemia & $26(96 \%)$ \\
\hline Grade 3 & $3(11 \%)$ \\
\hline Grade 4 & $0(0 \%)$ \\
\hline Elevated creatinine & $11(41 \%)$ \\
\hline Grade 3 & 0 \\
\hline Grade 4 & 0 \\
\hline Skin toxicity & $6(22 \%)$ \\
\hline Grade 3 & 0 \\
\hline Grade 4 & 0 \\
\hline Other toxicity & $16(59 \%)$ \\
\hline \multicolumn{2}{|c|}{ Postoperative complications } \\
\hline Acute kidney failure & 1 \\
\hline Cerebellitis & 1 \\
\hline Anastomotic leakage & 1 \\
\hline Burst abdomen & 1 \\
\hline \multicolumn{2}{|c|}{ Chemotherapy and radiotherapy } \\
\hline Nausea & 7 \\
\hline Neutropenic fever & 3 \\
\hline Gastritis & 3 \\
\hline Diarrhea & 1 \\
\hline Constipation & 1 \\
\hline
\end{tabular}


Urinary tract infection

1

Pulmonary embolism

1

Hyperkalemia

\section{Table 3: Treatment toxicity}

(4) 


\begin{tabular}{|c|c|c|c|c|c|}
\hline Sex & $\mathrm{n}$ & ARFS & DMFS & DFS & OAS \\
\hline Male & 14 & $66.6 \% p=0.39$ & $60.1 \% p=0.91$ & $36.4 \% p=0.61$ & $48.8 \% p=0.32$ \\
\hline Female & 13 & $85.7 \%$ & $75.5 \%$ & $62.9 \%$ & $72.9 \%$ \\
\hline \multicolumn{6}{|l|}{ Age } \\
\hline$<60$ years & 11 & $87.5 \% p=0.12$ & $51.9 \% p=0.13$ & $41.6 \% p=0.67$ & $53.0 \% p=0.76$ \\
\hline$\geq 60$ years & 16 & $64.3 \%$ & $80.0 \%$ & $54.8 \%$ & $66.2 \%$ \\
\hline \multicolumn{6}{|l|}{ Histology } \\
\hline Liposarcoma & 13 & $75.8 \% p=0.91$ & $58.6 \% p=0.52$ & $39.9 \% p=0.57$ & $57.5 \% p=0.78$ \\
\hline Other sarcoma & 14 & $72.7 \%$ & $77.4 \%$ & $58.0 \%$ & $62.3 \%$ \\
\hline \multicolumn{6}{|l|}{ Tumor diameter } \\
\hline$<100 \mathrm{~mm}$ & 13 & $85.7 \% p=0.08$ & $56.3 \% p=0.46$ & $43.8 \% p=0.67$ & $61.9 \% p=0.50$ \\
\hline$\geq 100 \mathrm{~mm}$ & 14 & $64.5 \%$ & $78.6 \%$ & $52.4 \%$ & $58.6 \%$ \\
\hline \multicolumn{6}{|l|}{ Primary vs. Recurrent } \\
\hline Primary & 18 & $72.2 \% p=0.32$ & $73.1 \% p=0.35$ & $52.2 \% p=0.87$ & $64.5 \% p=0.75$ \\
\hline Recurrent & 9 & $80.0 \%$ & $55.6 \%$ & $41.7 \%$ & $53.3 \%$ \\
\hline \multicolumn{6}{|l|}{ Chemotherapy given } \\
\hline$<2$ cycles & 25 & $50 \% * p=0.42$ & $50 \% * p=0.92$ & $0 \% * p=0.59$ & $50 \% * p=0.80$ \\
\hline$\geq 2$ cycles & 2 & $74.8 \%$ & $65.3 \%$ & $48.1 \%$ & $63.1 \%$ \\
\hline \multicolumn{6}{|l|}{ Radiotherapy method } \\
\hline $3 \mathrm{D}$ & 19 & $79.4 \% p=0.53$ & $60.8 \% p=0.20$ & $49.0 \% p=0.56$ & $61.1 \% p=0.93$ \\
\hline Intensity-modulated & 8 & $68.6 \%$ & $75.0 \%$ & $28.6 \%$ & $55.6 \%$ \\
\hline \multicolumn{6}{|l|}{ Treatment delay } \\
\hline$<5 d$ & 23 & $71.2 \% p=0.33$ & $77.2 \% p=0.36$ & $54.0 \% p=0.55$ & $67.7 \% p=0.11$ \\
\hline$>5 d$ & 4 & $100 \%$ & $25.0 \%$ & $25.0 \%$ & $25.0 \%$ \\
\hline \multicolumn{6}{|l|}{ Hyperthermia } \\
\hline$<5$ sessions & 8 & $85.6 \% p=0.25$ & $65.0 \% p=0.67$ & $51.7 \% p=0.37$ & $51.3 \% p=0.34$ \\
\hline$\geq 5$ sessions & 7 & $53.6 \%$ & $71.4 \%$ & $38.1 \%$ & $83.3 \%$ \\
\hline Grading & & & & & \\
\hline
\end{tabular}




\begin{tabular}{|llllll|} 
G2 & 12 & $80.8 \% p=0.74$ & $64.8 \% p=0.60$ & $55.6 \% p=0.56$ & $72.7 \% p=0.25$ \\
G3 & 14 & $78.6 \%$ & $67.7 \%$ & $44.8 \%$ & $42.3 \%$ \\
\hline
\end{tabular}

* = follow-up was not long enough

Table 4: Outcome and prognostic factors

\section{Figures}

\section{Radiotherapy
1.8 Gy OD}

Adriamycin
$(50 \mathrm{mg} / \mathrm{m} 2 / \mathrm{d})$

Ifosfamide

$(1.5 \mathrm{~g} / \mathrm{m} 2 / \mathrm{d})$

Hyperthermia (optional) $\sqrt{2}$

पIVt

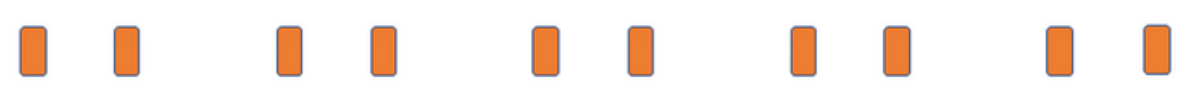

IIt

$\begin{array}{lll}3 & 4 & 5\end{array}$
6

\section{Figure 1}

Therapy regimen for neoadjuvant concurrent chemoradiotherapy \pm hyperthermia in retroperitoneal and intra-abdominal sarcoma.

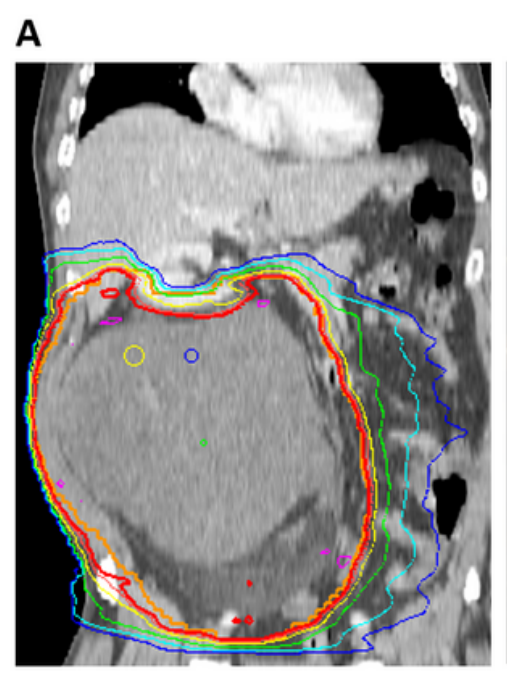

B

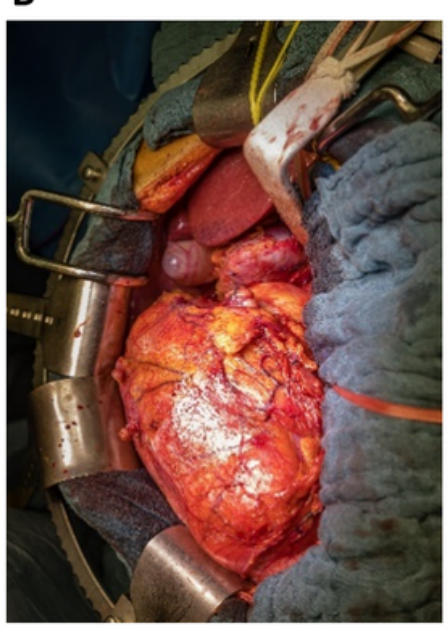

C

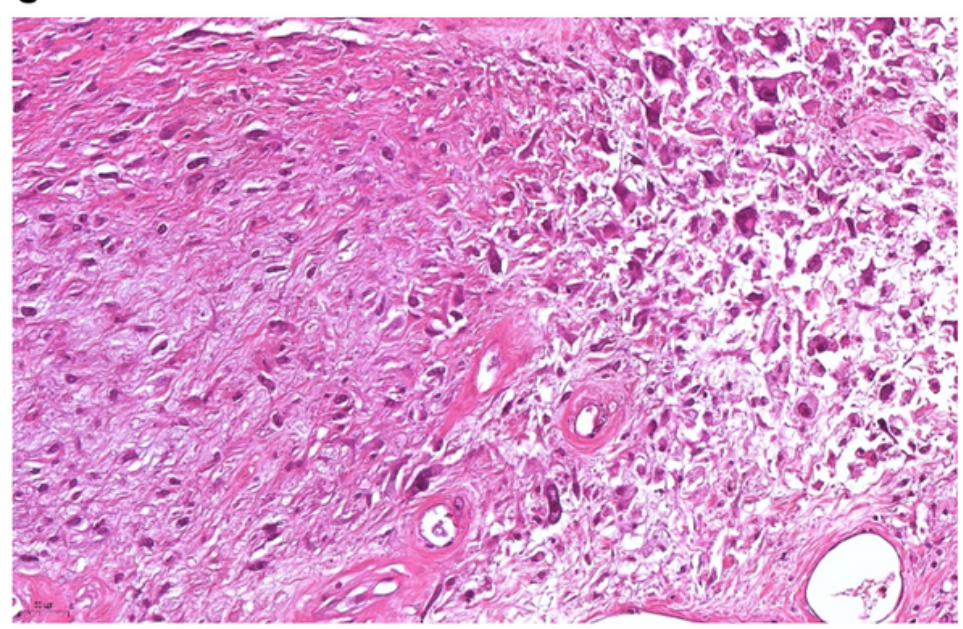

Figure 2 
Intra-abdominal liposarcoma with dose distribution of kidney-sparing radiotherapy (A), surgical site of enblock resection (B); strongly regressive altered residual tumor tissue after chemoradiotherapy (C)

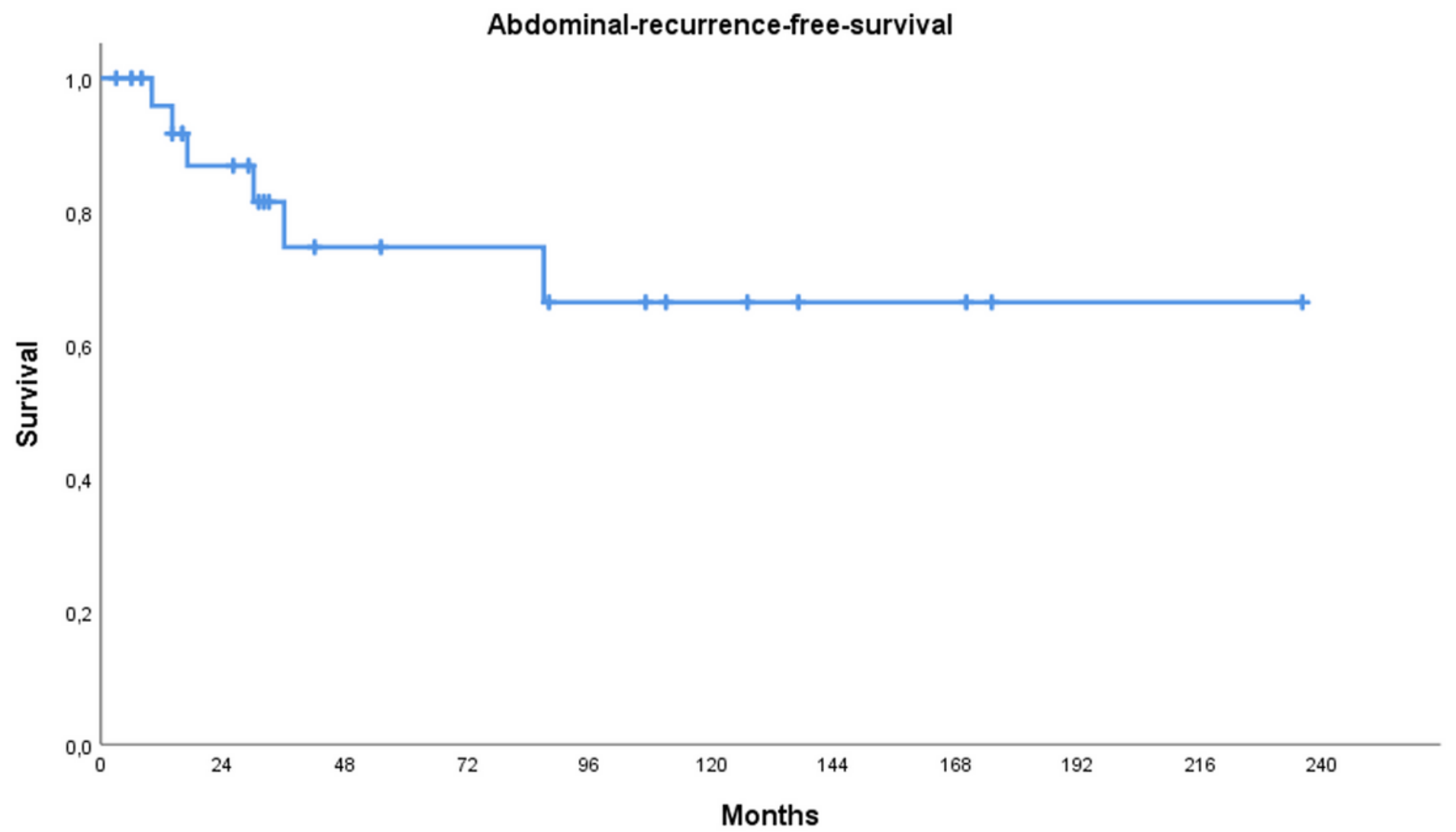

Figure 3

Abdominal recurrence free survival in months 


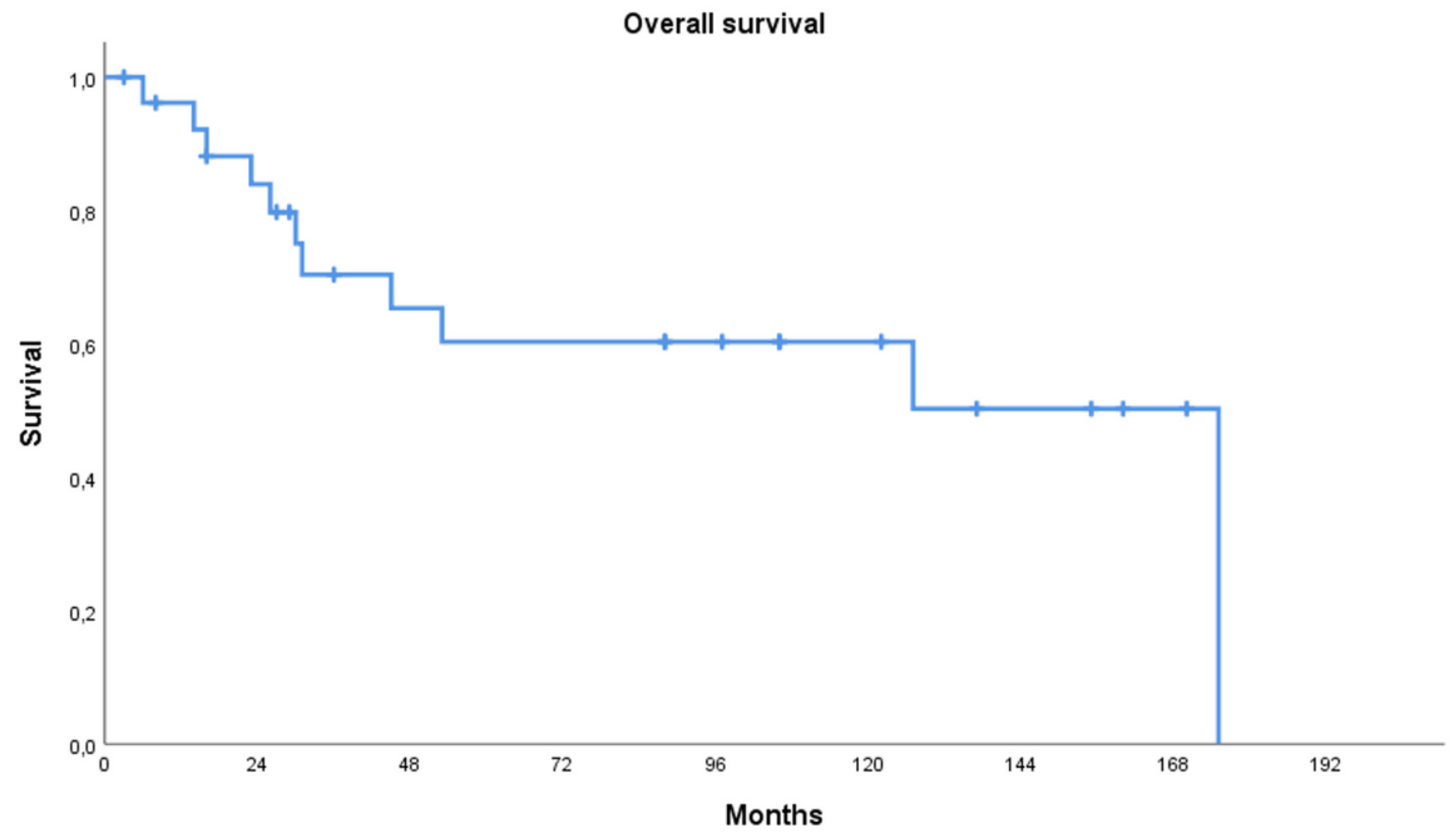

Figure 4

Overall survival in months 